\title{
Interleukin in endometriosis-associated infertility-pelvic pain: systematic review and meta-analysis
}

\author{
Helena Malvezzi, Camila Hernandes, Carla A Piccinato and Sérgio Podgaec \\ Hospital Israelita Albert Einstein, São Paulo, São Paulo, Brazil \\ Correspondence should be addressed to H Malvezzi; Email: helenamalvezzi@gmail.com
}

\begin{abstract}
The objective is to study the significance of altered interleukin levels in endometriosis-related infertility or pelvic pain. The present systematic review and meta-analysis includes a discussion on the roles of interleukin in the physiopathology of endometriosisassociated infertility and/or pelvic pain. We included all studies in which interleukins in peritoneal fluid, follicular fluid or serum from patients were measured and that correlated the findings with either peritoneal or deep endometriosis-associated infertility or pelvic pain. For the meta-analysis, we selected studies on the following cytokines: interleukin-1 (IL-1), interleukin-6 (IL-6) and interleukin-8 (IL-8). Endometriosis is a chronic inflammatory disease. Inflammatory processes clearly participate in the etiology of endometriosis. Cytokines are mediators of inflammation, and increase in their concentration in plasma or other body fluids signals the presence and extent of tissue lesions. A number of studies have reported on the association between higher cytokine levels and progression or maintenance of endometriosis and coexisting infertility or pelvic pain. The results of the analyses support that an association exists between elevated serum IL-6 and/or IL-8 concentrations and the occurrence of endometriosis-associated infertility. Such association was not found for endometriosis-associated pain. In spite of accumulated evidence on the association of proinflammatory cytokines and endometriosis, it still is not clear if and how these mediators participate in the physiopathology of endometriosis-associated infertility or pelvic pain, in part due to poor quality of the evidence established in the vast majority of interleukins and challenges in endometriosis research reproducibility. In summary, the results of the analyses support that an association exists between elevated serum IL-6 and/or IL-8 concentrations and the occurrence of endometriosis-associated infertility. Reproduction (2019) 158 1-12
\end{abstract}

\section{Introduction}

Endometriosis is a chronic inflammatory disease characterized by the presence of endometrial tissue outside of the uterine cavity, mainly attached to the peritoneum, pelvic organs or ligaments (Vercellini et al. 2014). The prevalence of endometriosis is assumed to be $10 \%$ of women in reproductive age (Kvaskoff et al. 2015). However, due to difficulties in understanding the diversity of the lesion's phenotypes, clinical symptoms and, in image diagnosis, the actual disease prevalence is unknown. The diagnostic difficulties frequently make endometriosis treatment a challenge. Nonetheless, untreated endometriosis can cause low quality of life, depression, inability to work, sexual dysfunction, infertility or diverse pain symptoms, including dysmenorrhea, deep dyspareunia and chronic pelvic pain (Carvalho et al. 2013). Although the factors determining the most common symptoms, such as infertility and pelvic pain, which affect respectively $40 \%$ (Eisenberg et al. 2018) and 50\% (Andres et al. 2014) of the patients have not been all identified, it is clear that inflammatory processes participate in the etiology of endometriosis (Jiang et al. 2016).
Relative to the causes of endometriosis-associated infertility, inflammatory mediators found in peritoneal and follicular fluids can act on cell proliferation, differentiation and blastocyst invasion. Among the described noxious effects are diminished oocyte quality (Da Broi \& Navarro 2016, Malvezzi et al. 2018); sperm/ oocyte interaction disorders (Hsu et al. 2015); embryo quality and its implantation and development (Stilley et al. 2012). Any of these effects leads to an altered reproductive condition (Haydardedeoglu \& Zeyneloglu 2015). Pain symptoms are also aggravated by the local inflammatory response secondary to the activation of immune responses (Arnold et al. 2013).

Cells involved in the immune response such as monocytes, macrophages, natural killer cells, $\mathrm{T}$ and $\mathrm{B}$ cells, regulatory $\mathrm{T}$ cells, as well as secreted cytokines and chemokines, participate in inflammation. Their role in endometriosis has been extensively studied, as cell numbers and cytokine concentrations were higher or altered in the peritoneal fluid of the endometriosis patients (Nesbitt-Hawes \& Ledger 2004, Podgaec et al. 2012, Gupta et al. 2016, Nisenblat et al. 2016).

Those cells, and also the ectopic endometrial implants and mesothelial cells of the peritoneum, 
synthesize and secrete cytokines, many of which were found to be related to endometriosis. Interleukin-1 (IL1), a stimulant of prostaglandins' synthesis (important pain and fever mediators), is increased in peritoneal fluid of endometriosis patients (Akoum et al. 2008). Interleukin-6 (IL-6) is a pleiotropic cytokine also secreted by macrophages that promotes endometrial cell proliferation and angiogenesis in endometriosis (Symons et al. 2018). Interleukin-8 (IL-8) is a potent angiogenic, pro-inflammatory and cellular proliferation cytokine, which was also found in the peritoneal fluid and facilitates endometrial cell adhesion (Sikora et al. 2017). Interleukin-10 may contribute to the altered immune regulation observed in endometriosis by limiting and terminating local inflammation and also leading to the removal of apoptotic cells which will reduce the local inflammatory response (Suen et al. 2014). It was reported to be elevated in serum from endometriosis stage III and IV patients (Suen et al. 2014).

Other cytokines such as IL-2, IL-4, IL-11, IL-12, IL-13, IL-15, IL-17, IL-18, IL-23 and IL-33 have also been associated with endometriosis. IL-4, IL-12, IL-15, IL-18, IL-23 and IL-33 were found to be elevated in endometriosis in the majority of studies, whereas IL-2 and IL-17 were downregulated or were not different from controls (McLaren et al. 1997, Furuya et al. 2003, Arici et al. 2003a,b, Zhang et al. 2005, Mikolajczyk et al. 2006, Fairbanks et al. 2009, Andreoli et al. 2011, Santulli et al. 2012).

Considering the controversial amount of information provided, the objective of this systematic review was to conduct a critical survey of the published papers reporting on interleukins quantified in serum, peritoneal fluid (PF) or follicular fluid (FF) in endometriosisassociated infertility and/or endometriosis-associated pelvic pain and to perform a meta-analysis of the papers whose data fulfilled the requirements for such analysis.

\section{Methods}

This study was designed according to the PRISMA statement for systematic reviews (Supplementary Fig. 1, see section on supplementary data given at the end of this article), and it was carried out at Hospital Israelita Albert Einstein in São Paulo, Brazil. The focal point keywords were 'interleukins', 'endometriosis', 'pelvic pain' and 'infertility', but we also included different keywords for a more complete combination of results. The search method was applied to PubMed, Medline, EMBASE, Scopus and Lilacs databases (the last search was performed on 1 June, 2018). The search builders are shown in Supplementary Fig. 2.

For meta-analysis, we selected studies that had welldescribed result data and only articles that had results expressed by measures of central tendency, variability and $P$ values or, at least, by measures of central tendency and $P$ values clearly depicted in figures, graphs or in text. Meta-analysis was carried out in the following conditions: (a) a minimum of two studies with information on the outcome of interest and effect size in the same direction; (b) a minimum of three studies, regardless of the direction of effect estimation (Valentine et al. 2010). Random effect models were considered and effect sizes (standardized mean differences) were estimated with a 95\% confidence interval. Absence of difference between groups was determined when $P$ values were greater than 0.05 or when the $95 \%$ confidence interval contained zero. The I2 index and Cochran $\mathrm{Q}$ test were used to measure the heterogeneity of the different studies. After reviewing all selected papers, the ones that evaluated IL-1 and/or IL-6 and/or IL-8 in relation to endometriosis presented enough data for meta-analysis.

The heat map (Fig. 1) represents a compilation of results from all published articles stipulated by values ranging from -1 to 1 for every article result according to the interleukin evaluated. To each article a score was given as follows: one (1), when the IL concentrations were significantly higher $(P \leq 0.05)$ in the group of endometriosis patients compared to the control group; zero (0) when the difference in IL concentrations between these groups was not statistically significant $(P>0.05)$; minus one $(-1)$ when IL concentrations were significantly higher $(P \leq 0.05)$ in the control group compared to endometriosis patients. Colors were chosen considering the sum of the results; vivid red for higher score, which denotes more articles reporting significantly increased IL concentrations in endometriosis fluids compared to control patients and blue for articles reporting significantly lower IL concentrations in endometriosis patients versus controls.

Quality evaluation of included studies was performed by the methodological index for non-randomized studies - MINORS - (Fig. 2) and followed the literature scope to characterize the risk of bias in the compilation of articles selected for this review (Slim et al. 2003).

MINORS is a verified scoring tool for non-randomized studies and includes a 12-item assessment. The score of each item ranges from -1 (not reported), 0 (reported but inadequate) to 1 (reported and adequate); a question mark (?) is used when evaluation was not possible.

\section{Selection and exclusion criteria}

All studies that associated IL with endometriosis-associated infertility or pelvic pain were included. No data limits were imposed. Two reviewers performed eligibility assessment independently in an unblinded standardized manner. Disagreement on study inclusion was solved by consensus. A systematic review was performed analyzing the number of patients involved in the study, type of study, results and conclusions of each study, which interleukins were evaluated, association with infertility and/or pelvic pain. To be included in the systematic review, the study had to present data on interleukin concentrations in serum, PF or FF samples from endometriosis patients and had to compare the findings with the occurrence of peritoneal or deep endometriosis-associated infertility or pelvic pain.

\section{Results}

A total of 426 articles were retrieved through database searching: 172 from PubMed/Medline, 206 from EMBASE, 48 from Scopus and 0 from Lilacs. From 
$\underline{A}$

\begin{tabular}{l|ccc}
\hline & PF & FF & S \\
\hline IL-1 & 3 & 2 & 2 \\
IL-2 & -1 & 1 & 1 \\
IL-4 & -1 & 1 & 1 \\
IL-6 & 0 & 3 & 2 \\
IL-8 & 2 & 1 & 1 \\
IL-10 & 0 & 1 & 1 \\
IL-12 & 0 & 1 & 2 \\
IL-15 & 1 & 0 & $\mathrm{~nm}$ \\
IL-17 & 0 & $\mathrm{~nm}$ & 0 \\
IL-18 & 2 & 0 & 0 \\
IL-23 & 1 & $\mathrm{~nm}$ & 0 \\
\hline
\end{tabular}

B

\begin{tabular}{c|cc}
\hline \multicolumn{1}{c}{} & PF & S \\
\hline IL-1 & -1 & $\mathrm{~nm}$ \\
IL-2 & 1 & $\mathrm{~nm}$ \\
IL-4 & 1 & $\mathrm{~nm}$ \\
IL-6 & 2 & $\mathrm{~nm}$ \\
IL-8 & 0 & 0 \\
IL-18 & 2 & $\mathrm{~nm}$ \\
IL-33 & 1 & $\mathrm{~nm}$ \\
\hline
\end{tabular}

Figure 1 Values range from vivid red for higher number of articles demonstrating significant increased interleukin (IL) concentration in endometriosis fluids compared to control patients to blue for significant decreased IL concentration in endometriosis. This table is a compilation of articles results, where to each article a score was given, being 1 for positive significance, 0 for non-significant and -1 for negative significance in IL concentration in endometriosis patients compared to control. For a complete description of results see Tables 1, 2, 3, 4, 5 and 6. (A) 3.1. Interleukins and infertility in endometriosis patients: IL-1: PF $=3$ studies $>; 4$ studies $n s ; F F=2$ studies $>; 2$ studies ns; $S=2$ studies $>; 5$ studies ns. IL-2: $\mathrm{PF}=1$ studies $<; 1$ study $\mathrm{ns} ; \mathrm{FF}=1$ study $>; \mathrm{S}=$ study $1>$. IL-4: $\mathrm{PF}=1$ study<; $1 \mathrm{~ns} ; \mathrm{FF}=1>; \mathrm{S}=1$ study $>$. IL-6: $\mathrm{PF}=2$ studies $>; 2<; 3$ studies ns; $\mathrm{FF}=3$ studies $>; 3$ studies $\mathrm{ns} ; \mathrm{S}=3$ studies $>; 1$ study $<; 1$ study ns; IL-8: PF $=2$ studies $>; 1$ study ns; $\mathrm{FF}=1$ study $>; 2$ studies ns; $\mathrm{S}=1$ study $>; 2$ studies ns. IL-10: $\mathrm{PF}=3$ studies $n s ; F F=1$ study $>; S=1$ study $>; 1$ study ns. IL-12: $P F=3$ studies $n s ; F F=1$ study $>; S=2$ studies $>$. IL-15: $P F=1$ study $>; F F=1$ study ns. IL-17: $P F=2$ studies $n s ; S=1$ study ns. IL-18: PF $=2$ studies $>; 1$ study $n s ; F F=1$ study $n s ; S=3$ studies $n s$. IL-23: $\mathrm{PF}=1$ study $>; S=1$ study ns. (B) Interleukins associated with pelvic pain in endometriosis patients: IL-1 $\beta: \mathrm{PF}=<; 1 \mathrm{study} n \mathrm{~ns}$ IL-2: PF $=1$ study $>$. IL-4: $\mathrm{PF}=1$ study $>$. IL-6: $\mathrm{PF}=2$ studies $>; 1$ study ns. IL-8: $\mathrm{PF}=1$ study ns; $\mathrm{S}=1$ study ns. IL-18: $\mathrm{PF}=2$ studies $>$. IL-33: PF $=1$ study $>$. $\mathrm{PF}=$ peritoneal fluid; $\mathrm{FF}=$ follicular fluid; $\mathrm{S}=$ serum; $>=$ higher IL concentration in endometriosis compared to control; $\mathrm{ns}=$ non-significant IL difference between endometriosis and control; <lower IL concentration in endometriosis compared to control; nm = not measured.

this list, all duplicates were removed resulting in 218 articles. After eliminating reviews and articles written in languages other than English or Portuguese, the final list consisted of 167 articles. Abstracts and references of all studies identified by the search strategy were examined and 67 relevant papers were read in full. All papers excluded from the review and the respective reasons for it appear in Supplementary Table 1. Finally, as shown in Supplementary Fig. 1, 26 studies fulfilled the selection criteria and were included in the systematic review. For meta-analysis, eight studies were selected; in fact, when considering different interleukins and fluids, the number of studies with available data ranges between 2 and 4 . The heat map, quality score and baseline characteristics of included studies are shown in Figs 1 and 2 and Tables 1, 2, 3, 4, 5 and 6.

\section{Interleukins and infertility in endometriosis patients}

Interleukin-1 (IL-1, IL-1 $\alpha$ and IL-1 $\beta$ )

IL-1, which includes the related cytokines IL- $1 \alpha$ and IL-1 $\beta$, exerts strong pro-inflammatory activities. In endometriosis, increased IL-1 levels have been associated with major symptoms; however, the association between IL-1 levels in serum, FF and PF and endometriosisassociated infertility is not evident (Table 1). For instance, five out of ten eligible articles belonging to our systematic review found no significant differences between FF, PF and serum IL-1 concentrations in endometriosis infertile patients compared to the respective control groups (Ueki et al. 1994, Pellicer et al. 1998, Oku et al. 2004, Skrzypczak et al. 2005, Wunder et al. 2006).

Regarding PF IL-1, meta-analysis information was available in two studies: one reported higher values for the endometriosis-associated infertility group (SMD $2.48,95 \% \mathrm{Cl} 1.81-3.14$ ), while in the other, there was no difference between groups (SMD, $-0.06 ; 95 \% \mathrm{Cl}$, -0.63 to 0.52$)$. Since there are only two non-concordant effects studies, the estimate combined effect was not determined. The $l^{2}$ value was $96.88 \%(95 \% \mathrm{Cl} 84.32$ to $>99.9 \%$ ), indicating a high degree of heterogeneity. Moreover, one study quantified PF IL-1 in endometriosis but IL-1 was undetected in control patients (KonderaAnasz et al. 2005). Additionally, in PF of infertile women with endometriosis, IL-1 was quantified as significantly higher than control patients (Akoum et al. 2008, Michaud et al. 2011).

An interesting result compared different stages of endometriosis. On one hand, PF IL-1 was higher in early stages of the disease (Akoum et al. 2008), whereas on the other hand, advanced stages presented higher PF and serum IL-1 (Kondera-Anasz et al. 2005).

IL-1 information on FF was available for three studies. The SMD between women with endometriosisassociated infertility and controls was estimated at 2.18 $(95 \% \mathrm{Cl}-0.04$ to $4.40, P$ 0.054) showing that there was not sufficient evidence that FF IL-1 values were increased among women with endometriosis and were 


\begin{tabular}{|c|c|c|c|c|c|c|c|c|c|c|c|c|}
\hline & 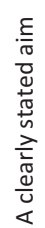 & 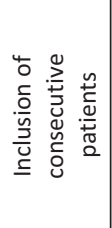 & 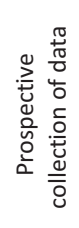 & 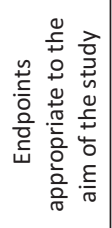 & 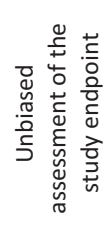 & 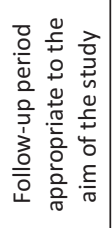 & 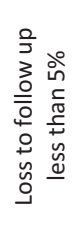 & 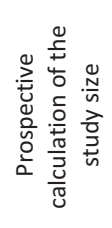 & 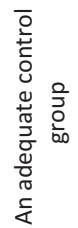 & 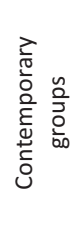 & 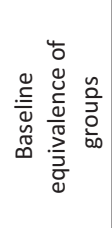 & 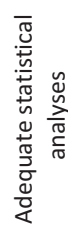 \\
\hline Altered expression of & 1 & 1 & 1 & 1 & -1 & ? & -1 & -1 & ? & 1 & -1 & 1 \\
\hline Changes of cytokines & 0 & 1 & 1 & 0 & -1 & $?$ & 1 & -1 & $?$ & 1 & -1 & 1 \\
\hline Concentrations of int & 1 & 1 & 1 & 1 & -1 & $?$ & 1 & -1 & $?$ & 1 & -1 & 1 \\
\hline Decreased & 1 & 1 & 1 & 0 & -1 & $?$ & 1 & -1 & $?$ & 1 & -1 & 1 \\
\hline Detection of & 1 & 1 & 1 & 0 & -1 & $?$ & 1 & -1 & $?$ & 1 & -1 & 1 \\
\hline Imbalance in the & 1 & 1 & 1 & 1 & -1 & $?$ & 1 & -1 & $?$ & 1 & 1 & 1 \\
\hline Immunological and & 1 & 1 & 1 & 0 & -1 & $?$ & 1 & -1 & $?$ & -1 & -1 & 1 \\
\hline Increased ENA-78 in & 1 & 1 & 1 & 1 & -1 & $?$ & 1 & -1 & $?$ & 1 & -1 & 1 \\
\hline Increased interleukin- & 1 & 1 & 1 & 1 & -1 & $?$ & 1 & -1 & $?$ & 1 & 1 & 1 \\
\hline Increased levels of & 1 & 1 & 1 & 1 & -1 & $?$ & 1 & -1 & $?$ & 1 & -1 & 1 \\
\hline Interleukin-6 and & 1 & 1 & 1 & 1 & -1 & $?$ & 1 & -1 & $?$ & 1 & -1 & 1 \\
\hline Interleukin-8 serum & 1 & 1 & 1 & 1 & -1 & $?$ & 1 & -1 & $?$ & 1 & 0 & 1 \\
\hline Intrafollicular & 1 & 1 & 1 & 1 & 1 & $?$ & 1 & 1 & $?$ & 1 & 1 & 1 \\
\hline Intrafollicular & 1 & 1 & 1 & 1 & -1 & $?$ & 1 & -1 & $?$ & 1 & -1 & 1 \\
\hline Macrophage & 0 & 1 & 1 & 0 & -1 & $?$ & 1 & -1 & $?$ & 1 & -1 & 1 \\
\hline Serum and peritoneal & 1 & 1 & 1 & 1 & -1 & $?$ & 1 & -1 & $?$ & 1 & -1 & 1 \\
\hline Peritoneal and serum & 1 & 1 & 1 & 1 & -1 & $?$ & 1 & -1 & $?$ & 1 & -1 & 1 \\
\hline Peritoneal fluid & 1 & 1 & 1 & 1 & -1 & $?$ & 1 & -1 & $?$ & 1 & -1 & 1 \\
\hline Peritoneal fluid & 1 & 1 & 1 & 1 & -1 & $?$ & 1 & -1 & $?$ & 1 & -1 & 1 \\
\hline Peritoneal & 1 & 1 & 1 & 1 & -1 & $?$ & 1 & -1 & $?$ & 1 & 1 & 1 \\
\hline Peritoneal fluid & 1 & 1 & 1 & 1 & -1 & $?$ & 1 & -1 & $?$ & 1 & -1 & 1 \\
\hline Selected cytokines & 1 & 1 & 1 & 1 & -1 & ? & 1 & -1 & $?$ & 1 & 1 & 1 \\
\hline Serum and peritoneal & 1 & 1 & 1 & 1 & -1 & $?$ & 1 & -1 & $?$ & 1 & 1 & 1 \\
\hline Thelper (Th)1, Th2, & 1 & 1 & 1 & 1 & -1 & $?$ & 1 & -1 & $?$ & 1 & -1 & 1 \\
\hline The follicular and & 1 & 1 & 1 & 1 & -1 & $?$ & 1 & -1 & $?$ & 1 & -1 & 1 \\
\hline Role of IL-18 in & 1 & 1 & 1 & 1 & -1 & $?$ & 1 & -1 & $?$ & 1 & -1 & 1 \\
\hline
\end{tabular}

Figure 2 The quality scores of included studies by methodological index for non-randomized studies. The scored -1 represents: not reported, 0 : reported but inadequate; 1 : reported and adequate and ?: not evaluated.

also infertile. The $R^{2}$ and Q test values were $98.61 \%$ (95\% Cl 94.82 to $>99.9 \%$ ) and less than 0.0001 respectively, indicating a high degree of heterogeneity. Higher FF IL-1 concentrations were described in endometriosis compared to FF from non-endometriotic infertile patients (Singh et al. 2016, Wu et al. 2017).

Serum IL-1 information was available for three studies ( $\mathrm{SMD}-0.48,95 \% \mathrm{Cl}-3.92$ to $2.96 ; P=0.783$ ) showing that there is not sufficient evidence that serum IL-1 values are different between women with and without endometriosis. The $P^{2}$ value and $P$ value for the $\mathrm{Q}$ were $98.91 \%$ (95\% Cl 95.98 to $>99.9 \%$ ) and less than 0.0001 respectively, indicating a high degree of heterogeneity.

\section{Interleukins IL-2, IL-12, IL-4, IL-10}

Interleukins IL-2 and IL-12 are closely related to T helper 1 (Th1) activation, whereas IL-4 and IL-10 are related to $\mathrm{T}$ helper 2 (Th2) activation during immunological responses. Th1-type responses are generally considered as pro-inflammatory in which the cytokines IL-2 and
IFN- $\gamma$ are synthesized by Th1 lymphocytes and IL-12 mainly by dendritic cells. Th2-type responses are considered as anti-inflammatory; Th2 lymphocytes synthesize IL-4 and IL-10, but several other cells also secrete IL-10, a cytokine associated to a regulatory phenotype. Both types of $\mathrm{T}$ cell responses are activated and regulated during the immune responses (Raphael et al. 2015). Four studies reported on IL-2, IL-12, IL-4 or IL-10 concentrations in endometriosis-associated infertility patients; however, the results are diverse and we emphasize that different corporal fluids were used in these studies (Table 2) (Furuya et al. 2003, Andreoli et al. 2011, Drosdzol-Cop et al. 2012, Singh et al. 2016).

Whereas Singh et al. (2016) reported statistically higher IL-2, IL-12, IL-4 or IL-10 concentrations in both $\mathrm{FF}$ and serum of endometriosis patients compared to controls undergoing in vitro fertilization procedures, the study by Furuya et al. (2003) did not find differences in PF dosages of the same cytokines between infertile endometriosis patients and controls. Corroborating Furuya et al. (2003) data, other researchers found no 
Table 1 Interleukin-1 distribution among endometriosis-associated infertility and control patients.

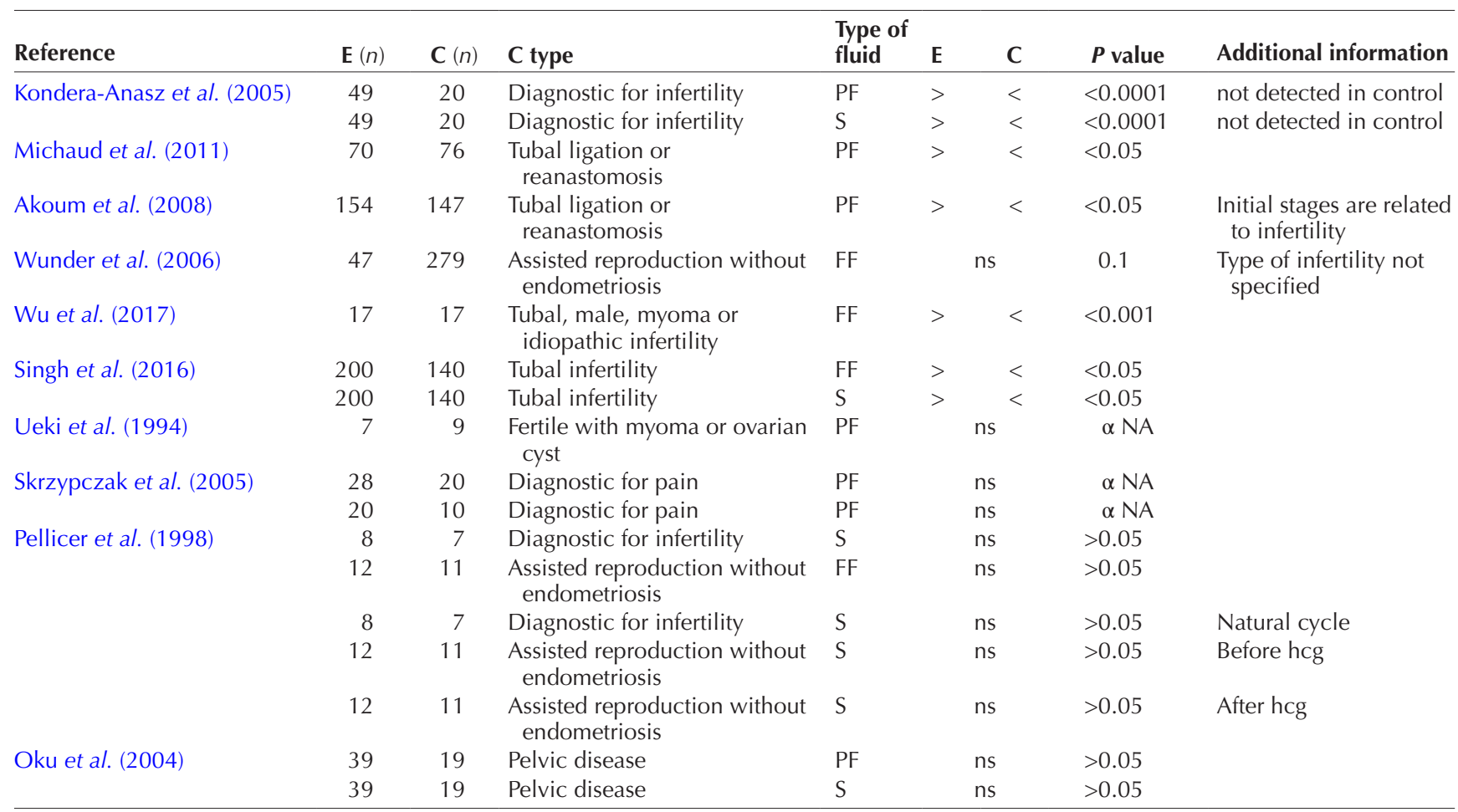

$\alpha \mathrm{NA}, \alpha$ error not available; C, control; E, endometriosis; FF, follicular fluid; ND, not detected; ns, non-significant; PF, peritoneal fluid; S, serum.

difference in PF and serum levels of IL-10 or IL-12 also between infertile endometriosis patients and controls (Andreoli et al. 2011). On the other hand, Drosdzol-Cop et al. (2012) reported higher IL-4 and low IL-2 levels in the serum and PF from endometriosis patients suggesting activation of a Th2 response (Drosdzol-Cop et al. 2012).

\section{Interleukin-6}

Despite being the most investigated interleukin in endometriosis, its relation with endometriosis-associated infertility is still unclear. IL-6 is a multifunctional cytokine involved in numerous immunological processes such as regulation of the immune response, inflammation,

Table 2 Interleukin-2, -4, -10 and -12 distribution among endometriosis-associated infertility and control patients.

\begin{tabular}{|c|c|c|c|c|c|c|c|c|c|}
\hline Author, year & $\mathbf{E}(n)$ & $\mathbf{C}(n)$ & C type & Type of fluid & IL & $\mathbf{E}$ & & C & $P$ value \\
\hline \multirow[t]{8}{*}{ Furuya et al. (2003) } & 14 & 3 & Pelvic inflammatory disease & PF & IL-2 & $<$ & & $>$ & $<0.05$ \\
\hline & 14 & 3 & Pelvic inflammatory disease & PF & IL-4 & $<$ & & $>$ & $<0.05$ \\
\hline & 14 & 3 & Pelvic inflammatory disease & PF & IL-10 & & ns & & $\alpha \mathrm{NA}$ \\
\hline & 14 & 3 & Pelvic inflammatory disease & PF & IL-12 & & $\mathrm{ns}$ & & $\alpha \mathrm{NA}$ \\
\hline & 14 & 12 & Benign ovarian cyst & PF & IL-2 & & $\mathrm{ns}$ & & $\alpha \mathrm{NA}$ \\
\hline & 14 & 12 & Benign ovarian cyst & PF & IL-4 & & ns & & $\alpha \mathrm{NA}$ \\
\hline & 14 & 12 & Benign ovarian cyst & PF & IL-10 & & ns & & $\alpha \mathrm{NA}$ \\
\hline & 14 & 12 & Benign ovarian cyst & PF & IL-12 & & ns & & $\alpha \mathrm{NA}$ \\
\hline \multirow[t]{8}{*}{ Singh et al. (2016) } & 200 & 140 & Tubal infertility & $\mathrm{FF}$ & IL-2 & $>$ & & $<$ & $<0.05$ \\
\hline & 200 & 140 & Tubal infertility & $\mathrm{FF}$ & IL-4 & $>$ & & $<$ & $<0.05$ \\
\hline & 200 & 140 & Tubal infertility & $\mathrm{FF}$ & IL-10 & $>$ & & $<$ & $<0.05$ \\
\hline & 200 & 140 & Tubal infertility & $\mathrm{FF}$ & IL-12 & $>$ & & $<$ & $<0.05$ \\
\hline & 200 & 140 & Tubal infertility & $S$ & IL-2 & $>$ & & $<$ & $<0.05$ \\
\hline & 200 & 140 & Tubal infertility & S & IL-4 & $>$ & & $<$ & $<0.05$ \\
\hline & 200 & 140 & Tubal infertility & $S$ & IL-10 & $>$ & & $<$ & $<0.05$ \\
\hline & 200 & 140 & Tubal infertility & $S$ & IL-12 & $>$ & & $<$ & $<0.05$ \\
\hline \multirow[t]{4}{*}{ Andreoli et al. (2011) } & 40 & 40 & Tubal ligation & PF & IL-10 & & $\mathrm{ns}$ & & 0.209 \\
\hline & 40 & 40 & Tubal ligation & PF & IL-12 & & ns & & 0.344 \\
\hline & 40 & 40 & Tubal ligation & $S$ & IL-10 & & $\mathrm{ns}$ & & 0.604 \\
\hline & 40 & 40 & Tubal ligation & S & IL-12 & & $\mathrm{ns}$ & & 0.203 \\
\hline
\end{tabular}

$\alpha \mathrm{NA}, \alpha$ error not available; C, control; E, endometriosis; FF, follicular fluid; IL, interleukin; ND, not detected; ns, non-significant; PF, peritoneal fluid; S, serum. 
Table 3 Interleukin-6 distribution among endometriosis-associated infertility and control patients.

\begin{tabular}{|c|c|c|c|c|c|c|c|c|c|}
\hline Author, year & $\mathbf{E}(n)$ & $\mathbf{C}(n)$ & C type & $\begin{array}{l}\text { Type of } \\
\text { fluid }\end{array}$ & E & & $\mathrm{C}$ & $P$ value & Additional information \\
\hline Liu et al. (2000) & 14 & 11 & $\begin{array}{l}\text { Diagnostic laparoscopy for } \\
\text { infertility }\end{array}$ & PF & $>$ & & $<$ & $<0.01$ & \\
\hline \multirow[t]{2}{*}{ Buyalos et al. (1992) } & 10 & 10 & Tubal infertility & $\mathrm{FF}$ & & Ns & & $\alpha \mathrm{NA}$ & \\
\hline & 10 & 10 & Antisperm antibody & FF & & Ns & & $\alpha \mathrm{NA}$ & \\
\hline \multirow[t]{2}{*}{ Furuya et al. (2003) } & 14 & 3 & Pelvic inflammatory disease & PF & $<$ & & $>$ & $<0.01$ & \\
\hline & 14 & 12 & Benign ovarian cyst & PF & $>$ & & $<$ & $<0.05$ & \\
\hline Wunder et al. (2006) & 47 & 279 & $\begin{array}{l}\text { Assisted reproduction without } \\
\text { endometriosis }\end{array}$ & $\mathrm{FF}$ & & ns & & 0.34 & \\
\hline Harada et al. (1997) & 19 & 19 & $\begin{array}{l}\text { Diagnostic laparoscopy for } \\
\text { infertility }\end{array}$ & $\mathrm{PF}$ & $<$ & & $>$ & $<0.05$ & \\
\hline Wu et al. (2017) & 17 & 17 & $\begin{array}{l}\text { Tubal, male, myoma or idiopathic } \\
\text { infertility }\end{array}$ & $\mathrm{FF}$ & $>$ & & $<$ & 0.01 & \\
\hline \multirow[t]{2}{*}{ Singh et al. (2016) } & 200 & 140 & Tubal infertility & $\mathrm{FF}$ & $>$ & & $<$ & $<0.05$ & \\
\hline & 200 & 140 & Tubal infertility & S & $>$ & & $<$ & $<0.05$ & \\
\hline Ueki et al. (1994) & 7 & 9 & Fertile with myoma or ovarian cyst & $\mathrm{PF}$ & & ns & & $\alpha \mathrm{NA}$ & \\
\hline \multirow[t]{2}{*}{ Skrzypczak et al. (2005) } & 28 & 20 & Infertile without endometriosis & PF & & ns & & $\alpha \mathrm{NA}$ & Infertile endometriosis \\
\hline & 20 & 10 & Fertile & PF & & ns & & $\alpha \mathrm{NA}$ & Fertile endometriosis \\
\hline \multirow[t]{5}{*}{ Pellicer et al. (1998) } & 8 & 7 & $\begin{array}{l}\text { Diagnostic laparoscopy for } \\
\text { infertility }\end{array}$ & S & $<$ & & $>$ & $<0.05$ & \\
\hline & 12 & 11 & $\begin{array}{l}\text { Assisted reproduction without } \\
\text { endometriosis }\end{array}$ & $\mathrm{FF}$ & $>$ & & $<$ & $<0.05$ & \\
\hline & 8 & 7 & $\begin{array}{l}\text { Diagnostic laparoscopy for } \\
\text { infertility }\end{array}$ & S & $>$ & & $<$ & $<0.05$ & Natural cycle \\
\hline & 12 & 11 & $\begin{array}{l}\text { Assisted reproduction without } \\
\text { endometriosis }\end{array}$ & S & $>$ & & $<$ & $<0.05$ & Before hcg \\
\hline & 12 & 11 & $\begin{array}{l}\text { Assisted reproduction without } \\
\text { endometriosis }\end{array}$ & $S$ & & ns & & $\alpha \mathrm{NA}$ & After hcg \\
\hline
\end{tabular}

$\alpha \mathrm{NA}, \alpha$ error not available; $\mathrm{C}$, control; E, endometriosis; FF, follicular fluid; ND, not detected; ns, non-significant; PF, peritoneal fluid; S, serum.

angiogenesis and pregnancy outcome. Several groups (Table 3) studied its production and association with infertility. Five out of nine studies failed to find any difference in PF IL-6 levels between endometriosis-associated infertility patients and controls (Ueki et al. 1994, Furuya et al. 2003), FF (Buyalos et al. 1992, Wunder et al. 2006) and also in serum (Drosdzol-Cop \& Skrzypulec-Plinta 2012). In four studies higher IL-6 concentrations in infertile endometriosis compared with non-endometriosis patients were found in the PF (Harada et al. 1997, Liu et al. 2000) and FF (Singh et al. 2016, Wu et al. 2017). One particular study (Pellicer et al. 1998) compared within the endometriosis patients group, those undergoing natural cycle with those with stimulated cycles, and also with controls (tubal factor controls). The authors found that in natural cycling women, serum IL-6 was higher in endometriosis compared to controls; during stimulated cycles, serum IL-6 decreased in endometriosis patients and increased just after human chorionic hormone administration; FF IL-6 was also higher in endometriosis. Other authors reported that when fertile women with or without endometriosis were compared with infertile endometriosis patients, IL-6 concentrations were significantly higher in the fertile group $(P<0.05)$ (Skrzypczak et al. 2005).

Table 4 Interleukin-8 distribution among endometriosis-associated infertility and control patients.

\begin{tabular}{|c|c|c|c|c|c|c|c|c|c|}
\hline Author, year & $\mathbf{E}(n)$ & $\mathbf{C}(n)$ & C type & $\begin{array}{l}\text { Type of } \\
\text { fluid }\end{array}$ & $\mathbf{E}$ & & C & $P$ value & Additional information \\
\hline Wunder et al. (2006) & 47 & 279 & $\begin{array}{l}\text { Assisted reproduction without } \\
\text { endometriosis }\end{array}$ & $\mathrm{FF}$ & & רs & & 0.63 & \\
\hline \multirow[t]{2}{*}{ Wu et al. (2017) } & 17 & 17 & $\begin{array}{l}\text { Tubal, male, myoma or idiopathic } \\
\text { infertility }\end{array}$ & FF & & ns & & $\alpha \mathrm{NA}$ & \\
\hline & 17 & 17 & $\begin{array}{l}\text { Tubal, male, myoma or idiopathic } \\
\text { infertility }\end{array}$ & S & & ns & & $\alpha \mathrm{NA}$ & \\
\hline \multirow[t]{2}{*}{ Singh et al. (2016) } & 200 & 140 & Tubal infertility & $\mathrm{FF}$ & $>$ & & $<$ & $<0.01$ & \\
\hline & 200 & 140 & Tubal infertility & S & $>$ & & $<$ & $<0.01$ & \\
\hline \multirow[t]{2}{*}{ Gazvani et al. (1998) } & 25 & 22 & $\begin{array}{l}\text { Diagnostic laparoscopy for pain or } \\
\text { infertility }\end{array}$ & $\mathrm{PF}$ & $>$ & & $<$ & 0.02 & \\
\hline & 25 & 22 & $\begin{array}{l}\text { Diagnostic laparoscopy for pain or } \\
\text { infertility }\end{array}$ & $S$ & & ns & & 0.27 & \\
\hline \multirow[t]{2}{*}{ Skrzypczak et al. (2005) } & 28 & 20 & Infertile without endometriosis & $\mathrm{PF}$ & $>$ & & $<$ & $<0.01$ & Infertile endometriosis \\
\hline & 20 & 10 & Fertile & $\mathrm{PF}$ & & ns & & $\alpha \mathrm{NA}$ & Fertile endometriosis \\
\hline
\end{tabular}

$\alpha \mathrm{NA}, \alpha$ error not available; C, control; E, endometriosis; FF, follicular fluid; ns, non-significant; PF, peritoneal fluid; S, serum. 
Table 5 Interleukin-15, -17,-18 and -23 distribution among endometriosis-associated infertility and control patients.

\begin{tabular}{|c|c|c|c|c|c|c|c|c|c|}
\hline Author, year & $\mathbf{E}(n)$ & $\mathbf{C}(n)$ & C type & Type of fluid & IL & $\mathbf{E}$ & & C & $P$ value \\
\hline \multirow[t]{2}{*}{ Arici et al. (2003a) } & 50 & 18 & Tubal ligation & PF & IL-18 & $>$ & & $<$ & 0005 \\
\hline & 47 & 279 & Assisted reproduction without endometriosis & $\mathrm{FF}$ & IL-15 & \multicolumn{3}{|c|}{$\mathrm{ns}$} & 0.78 \\
\hline Arici et al. (2003b) & 55 & 18 & Tubal ligation & PF & IL-15 & $>$ & & $<$ & $<0.001$ \\
\hline Velasco et al. (2010) & 84 & 24 & Ovarian cyst & OMA & IL-17 & \multicolumn{4}{|c|}{ ND } \\
\hline \multirow[t]{2}{*}{ Wu et al. (2017) } & 17 & 17 & Tubal, male, myoma or idiopathic infertility & $\mathrm{FF}$ & IL-18 & \multirow{2}{*}{\multicolumn{3}{|c|}{$\begin{array}{l}\mathrm{ns} \\
\mathrm{ns}\end{array}$}} & $\alpha \mathrm{NA}$ \\
\hline & 17 & 17 & Tubal, male, myoma or idiopathic infertility & S & IL-18 & & & & $\alpha \mathrm{NA}$ \\
\hline \multirow[t]{2}{*}{ Glitz et al. (2009) } & 34 & 22 & Tubal ligation & PF & IL-18 & \multicolumn{3}{|c|}{ ns } & $>0.05$ \\
\hline & 34 & 22 & Tubal ligation & $S$ & IL-18 & \multicolumn{3}{|c|}{$\mathrm{ns}$} & $>0.05$ \\
\hline Zhang et al. (2005) & 36 & 26 & Tubal ligation & PF & IL-17 & \multicolumn{3}{|c|}{ ns } & $>0.05$ \\
\hline \multirow[t]{4}{*}{ Andreoli et al. (2011) } & 40 & 40 & Tubal ligation & PF & IL-17 & \multicolumn{3}{|c|}{ ns } & 0.445 \\
\hline & 40 & 40 & Tubal ligation & $\mathrm{PF}$ & IL-23 & \multirow{3}{*}{\multicolumn{3}{|c|}{$\begin{array}{l}\mathrm{ns} \\
\mathrm{ns}\end{array}$}} & 0.006 \\
\hline & 40 & 40 & Tubal ligation & $\mathrm{S}$ & IL-17 & & & & 0.325 \\
\hline & 40 & 40 & Tubal ligation & $\mathrm{S}$ & IL-23 & & & & 0.209 \\
\hline \multirow[t]{2}{*}{ Oku et al. (2004) } & 39 & 19 & Pelvic disease & PF & IL-18 & \multirow[t]{2}{*}{$>$} & & $<$ & $<0.019$ \\
\hline & 39 & 19 & Pelvic disease & $\mathrm{S}$ & IL-18 & & רs & & 0.945 \\
\hline
\end{tabular}

$\alpha \mathrm{NA}, \alpha$ error not available; C, control; E, endometriosis; FF, follicular fluid; IL, interleukin; ND, not detected; ns, non-significant; PF, peritoneal fluid; S, serum.

IL-6 information in FF was available for meta-analysis in four studies (SMD $0.82,95 \% \mathrm{Cl}-0.60$ to $2.25, P=0.258$ ). The conclusion is that no sufficient evidence exists that FF IL-6 values are different between women with and without endometriosis. The $R^{2}$ and $\mathrm{Q}$ test values were $97.42 \%(95 \% \mathrm{Cl}$ of $91.45-99.82 \%)$ and less than 0.0001 respectively, indicating a high degree of heterogeneity, so that the estimation of effect size is inconclusive.

In the PF two studies were available; one had higher values for the endometriosis group (SMD 6.35, 95\% Cl: 4.42-8.28) and, in the other, no differences between groups were described (SMD 0.00, 95\% Cl: -0.57 to 0.57). Due to the non-concordant effect studies, the combined effect was not estimated. The $R^{2}$ value was $97.4 \%(95 \% \mathrm{Cl}$ of 86.9 to $>99.7 \%)$, indicating a high degree of heterogeneity.
On the other hand, two studies were available for serum IL-6, and both reported higher values in the endometriosis group (SMD 3.92, 95\% Cl 3.56-4.28, $P<0.001)$. In conclusion, even though coming from only two studies, there was sufficient evidence that serum IL-6 values are increased among women with endometriosis-associated infertility. The $R^{2}$ value was $0.0 \%(95 \% \mathrm{Cl}$ of $0.0-96.1 \%)$, indicating mild degree or absence of heterogeneity.

\section{Interleukin-8}

IL-8 is secreted by leukocytes, macrophages and endothelial cells stimulated, for instance, by IL-1 or TNF$\alpha$. It is a pro-inflammatory cytokine known to be a potent regulator of angiogenesis and a growth promoting factor (Long et al. 2016). Both properties may be important to

Table 6 Interleukin distribution among endometriosis-associated pain and control patients.

\begin{tabular}{|c|c|c|c|c|c|c|c|c|c|}
\hline Author, year & $\mathbf{E}(n)$ & $\mathbf{C}(n)$ & C type & $\begin{array}{l}\text { Type of } \\
\text { fluid }\end{array}$ & IL & $\mathbf{E}$ & C & $P$ value & $\begin{array}{l}\text { Additional } \\
\text { information }\end{array}$ \\
\hline Arici et al. (2003a) & 50 & 18 & Tubal ligation & PF & IL-18 & $>$ & $<$ & 0.005 & \\
\hline Michaud et al. (2011) & 70 & 76 & $\begin{array}{l}\text { Tubal ligation or } \\
\text { reanastomosis }\end{array}$ & PF & IL-1 $\beta$ & $P<0.05$ & & $<0.05$ & $\begin{array}{l}\text { No dispersion } \\
\text { values available }\end{array}$ \\
\hline \multirow[t]{2}{*}{ Velasco et al. (2010) } & 84 & 24 & Ovarian cyst & PF & IL-6 & $>$ & $<$ & $<0.01$ & \\
\hline & 84 & 24 & Ovarian cyst & PF & IL-8 & $\mathrm{ns}$ & $\alpha \mathrm{NA}$ & & \\
\hline $\begin{array}{l}\text { Manero \& Alcazar } \\
(2010)\end{array}$ & 26 & 25 & $\begin{array}{l}\text { Diagnostic laparoscopy } \\
\text { for pain }\end{array}$ & S & IL-8 & ns & $\alpha \mathrm{NA}$ & & \\
\hline Santulli et al. (2012) & 44 & 36 & $\begin{array}{l}\text { Diagnostic laparoscopy } \\
\text { for pain }\end{array}$ & PF & IL-33 & $>$ & $<$ & 0.013 & \\
\hline Rapkin et al. (2000) & 29 & 23 & $\begin{array}{l}\text { Pelvic adhesions or no } \\
\text { visible alteration }\end{array}$ & PF & IL-6 & ns & ${ }^{\alpha} \mathrm{NA}$ & $\begin{array}{r}\text { Compar } \\
\text { no pail }\end{array}$ & $\begin{array}{l}\text { between pain and } \\
\text { ong groups }\end{array}$ \\
\hline $\begin{array}{l}\text { Drosdzol-Cop \& } \\
\text { Skrzypulec-Plinta } \\
\text { (2012) }\end{array}$ & 33 & 11 & $\begin{array}{l}\text { Diagnostic laparoscopy } \\
\text { for pain }\end{array}$ & PF & IL-6 & $>$ & $<$ & 0.0002 & \\
\hline \multirow[t]{2}{*}{$\begin{array}{l}\text { Drosdzol-Cop et al. } \\
\text { (2012) }\end{array}$} & 33 & 17 & $\begin{array}{l}\text { Diagnostic laparoscopy } \\
\text { for pain }\end{array}$ & PF & IL-2 & $<$ & $>$ & 0.01 & \\
\hline & 33 & 17 & $\begin{array}{l}\text { Diagnostic laparoscopy } \\
\text { for pain }\end{array}$ & PF & IL-4 & $>$ & $<$ & 0.03 & \\
\hline \multirow[t]{2}{*}{ Oku et al. (2004) } & 39 & 19 & Pelvic disease & PF & IL-1 $\beta$ & ns & & $>0.05$ & \\
\hline & 39 & 19 & Pelvic disease & PF & IL-18 & $>$ & $<$ & $<0.019$ & \\
\hline
\end{tabular}

$\alpha \mathrm{NA}, \alpha$ error not available; C, control; E, endometriosis; FF, follicular fluid; IL, interleukin; ND, not detected; ns, non-significant; PF, peritoneal fluid; $S$, serum. 
endometriosis as they could possibly contribute to the inflammatory pathogenesis and infertility associated with the disease. The relationship between IL- 8 and infertility was reported in five published papers (Table 4) and all the studies that evaluated IL-8 in the PF found higher concentrations of this cytokine in endometriosis patients compared to control patients. Especially when comparing infertile women with and without endometriosis, IL-8 levels were higher in the endometriosis group (Gazvani et al. 1998, Skrzypczak et al. 2005).

The differences found for IL-8 PF levels, however, were not consistent among the studies that reported on FF and IL-8. Two groups found no statistically significant differences in IL-8 levels in FF (Wunder et al. 2006, Wu et al. 2017) and one study reported higher serum and FF IL-8 levels in infertile endometriosis patients compared to control patients enrolled in in vitro fertilization protocols (Singh et al. 2016).

Follicular and PF IL-8 data information was available in two studies. One of these studies reported higher values for the endometriosis group in the FF and in PF (SMD 9.27; 95\% Cl 9.54-10.0 for FF; 5.51, 95\% Cl 4.26-6.76 for PF) (Gazvani et al. 1998, Singh et al. 2016). The other study did not find any difference in IL-8 concentrations between endometriosis and control groups (SMD of $0.07,95 \% \mathrm{Cl}-0.24$ to 0.38 for $\mathrm{FF} ; 0.51,95 \% \mathrm{Cl}-0.07$ to 1.10 for PF) (Skrzypczak et al. 2005, Wunder et al. 2006). Therefore, no combined effect was estimated for FF and PF IL- 8 . The $R^{2}$ value was $99.8 \%(95 \% \mathrm{CI}$ 99.0 to $>99.9 \%$ ) and $98.0 \%(95 \% \mathrm{Cl} 90.0$ to $>99.8 \%)$ respectively, indicating a high degree of heterogeneity. For serum IL-8 both studies observed higher values in the endometriosis-associated infertility group (SMD $4.01,95 \% \mathrm{Cl} 3.45-4.33, P<0.001)$. In conclusion, there is sufficient analytical evidence that serum IL-8 values are increased in women with endometriosis-associated infertility. The $I^{2}$ value was $18.4 \%(95 \% \mathrm{Cl}$ of 0.0 to $>99 \%$ ), indicating a small degree of heterogeneity.

\section{Interleukin-15,-17,-18 and -23}

Endometriosis is widely known as an inflammatory condition, and IL-15, IL-17, IL-18 and IL-23 are proinflammatory cytokines that have been investigated in endometriosis (Table 5) (Musso et al. 1998, Oku et al. 2004, Lubberts 2015). Data on IL-23 appear in just one study and no difference was seen in serum and PF between endometriosis and control patients and no association of IL-23 was found with infertility (Andreoli et al. 2011). IL-23 is known to stimulate a Th17 response, but like IL-23, no difference in concentrations of IL-17 in PF or serum were found between endometriosis patients and controls (Zhang et al. 2005, Andreoli et al. 2011) and, in one study, neither was even detected in serum or PF (Velasco et al. 2010). Nevertheless, Zhang and collaborators reported significant difference in IL-17 levels between minimal/mild endometriosis versus moderate/severe stages of the disease and higher IL-17 concentrations in infertile versus fertile endometriosis patients (Zhang et al. 2005).

Lymphocytes and macrophages can secrete IL-15 and IL-18. PF of endometriosis patients contains higher numbers of macrophages (Santanam et al. 2002). Still, it is interesting to notice that the studies on IL-15 and IL-18 have conflicting results (Arici et al. 2003a,b, Oku et al. 2004, Wunder et al. 2006, Glitz et al. 2009, Wu et al. 2017).

While two groups found increased concentrations of IL-18 in PF of infertile endometriosis patients compared to controls, there was no association with infertility (Arici et al. 2003a,b, Oku et al. 2004). Another study found no difference in FF IL-18 between endometriosis and nonendometriosis patients (Wu et al. 2017) and still another research group did not find significant differences in PF IL-18 concentrations between the two groups of patients (Glitz et al. 2009).

With respect to IL-15, in one study, IL-15 was not detected either in FF or in serum from infertile women with endometriosis (Wu et al. 2017). In another study, no difference was observed in PF IL-15 (Wunder et al. 2006), and in a third study, PF IL-15 levels in endometriosis patients were higher compared to PF from controls. In this last study, PF IL-15 concentrations were found to be significantly different between minimal/mild endometriosis versus moderate/severe endometriosis. Similar differential result for PF IL-15 was also reported between deep endometriosis in comparison to endometrioma (Arici et al. 2003a,b).

\section{Interleukins and pelvic pain in endometriosis patients}

The association between IL-1, IL-2, IL-4, IL-6, IL-8, IL-18 or IL-33 and endometriosis-related pelvic pain was investigated by different research groups (Table 6) (Rapkin et al. 2000, Arici et al. 2003a,b, Oku et al. 2004, Manero \& Alcazar 2010, Velasco et al. 2010, Michaud et al. 2011, Drosdzol-Cop \& Skrzypulec-Plinta 2012, Drosdzol-Cop et al. 2012, Malhotra et al. 2012, Santulli et al. 2012).

The majority of these studies indicate an association between endometriosis-associated pain with cytokine concentration by reporting increased interleukin levels in PF from endometriosis patients compared to control participants. Only two of these studies report similar significant associations for serum cytokine levels in patients compared to controls (Drosdzol-Cop et al. 2012, Santulli et al. 2012).

Peritoneal fluid IL-1 and IL-18, IL-4 from PF and serum, and IL-33 from serum were augmented in all mentioned studies (Arici et al. 2003a,b, Oku et al. 2004, Michaud et al. 2011, Drosdzol-Cop et al. 2012, Santulli et al. 2012). However, positive correlation with dysmenorrhea $(r=0.270, P<0.05)$ and pelvic pain $(r=0.247, P<0.05)$ in endometriosis patients could only 
be established for IL-6 in one study (Velasco et al. 2010), and for IL-33 and dysmenorrhea also in just one study $(r=0.174 ; P=0.028)$ (Santulli et al. 2012).

Despite possibly being the most frequently studied interleukins in endometriosis, investigations on IL-6 and IL-8 led to inconsistent results. Some authors found significantly higher concentrations in endometriosis patients (Manero \& Alcazar 2010, Velasco et al. 2010, Drosdzol-Cop \& Skrzypulec-Plinta 2012), whereas others reported no difference in patients versus controls regardless of the evaluated fluid (Rapkin et al. 2000, Manero \& Alcazar 2010). The heat maps of endometriosis-associated pain IL can be seen in Fig. 1B.

\section{Discussion}

The present review and meta-analysis support the association between elevated serum pro-inflammatory interleukins, IL-6 and IL-8, and endometriosis-associated infertility. Similar studies failed to show the association of higher IL-6 and IL-8 levels with endometriosisassociated pain. In addition, the analysis of the included papers did not provide consistent results relative to differences in follicular or PF concentrations of any of the investigated cytokines (IL-6 and IL-8 included).

IL-1 studies, taken together, did not supply sufficient evidence of differences in concentration between endometriosis and control patients in the three fluids (serum, FF and PF).

Despite the small meta-analysis results, in accordance with other reviews (Harada et al. 2001, Arici 2002) our present analysis observed higher interleukin concentrations in infertile endometriosis patients compared to control participants, regardless of the inclusion criteria chosen for the control group (Fig. 1A and Tables 1, 2, 3, 4 and 5). The results for IL-1, IL-8, IL-10, IL-12, IL-15 and IL-23 concentrations in PF, FF and serum are either non-significant or higher in endometriosis. Regarding IL-2, IL-4 and IL-6 results from the analyzed studies are more diverse and in some of the studies, the IL concentrations reflect the menstrual cycle phase (Liu et al. 2000) or stage dependent (Skrzypczak et al. 2005).

The MINORS gives us the idea of how endometriosis research has been developed and, in that way, how challenging can its reproducibility and the robustness of its results given by poor quality of the evidence established be. Almost all selected papers (96\%) have bias in the assessment of study endpoints and have not calculated the study sample size prior to its beginning or, at least, it was not described. Another important point is the assessment of equivalence of groups and type of lesion, since the eligibility criteria for the control group and the presence, local and type of endometriosis described were very different among studies. Different hypotheses and endpoints require comparison with adequate control groups and, therefore, different results are expected. Some studies compared infertile endometriosis groups with fertile control groups, while others compared infertile endometriosis with infertile control or infertile versus fertile endometriosis.

When looking at the heat map (Fig. 1A), it is noticeable that the majority of positive results for higher concentration of ILs were found in the serum or FF from infertile endometriosis patients. On the other hand, results for PF are diverse, which could be due to patient or control group selection or to the cytokine-quantifying method (Fig. 2).

Apart from the results of the meta-analysis, the systematic review emphasizes an important fact: interleukin concentrations vary among infertile endometriosis patients depending on the menstrual cycle phase and endometriosis grade, and differences between studies depend on the chosen control group. Concerning endometriosis-associated pelvic pain, the results appear to be less conflicting. While looking at the heat map (Fig. 1A), the impression is that higher than control, IL concentrations tend to be reported more frequently for endometriosis patients in the existing studies. However, as the number of analyzable studies is still small, this assumption may be precocious. There is need for more studies on interleukin and chronic pelvic pain.

Since endometriosis is an inflammatory disease, the existing evidence suggests that infertility and pelvic pain associated with endometriosis result from the local peritoneal inflammatory reaction caused by the ectopic endometrial implants (Miller etal. 2017). After attachment to the peritoneum, the viability of the endometriotic focus may be maintained by local angiogenesis and secretion of growth factors and cytokines, mainly by peritoneal neutrophils and macrophages (Izumi et al. 2018). It is also known that macrophages and natural killer (NK) cells of patients with endometriosis may not eliminate all endometrial cells in the peritoneal cavity, which leads to aberrant cytokine secretion and inflammation (Izumi et al. 2018).

The presence of ectopic endometrium as well as cytokines and chemokines possibly recruit more cells (B and T lymphocytes, macrophages, NK cells, neutrophils), a cause of more inflammation. Cytokines are secreted by all these cell types and they activate the synthesis of other cytokines, inflammatory mediators such as prostaglandins and leukotrienes and chemokines. Chemokines are a family of secreted proteins that are chemotactic to a great variety of cells whose receptors are expressed upon stimulation on virtually all cell types. Interleukins and other cytokines either stimulate or downregulate cell proliferation and the synthesis of other cytokines and mediators in target cells, depending on the cytokine and target cell. Examples are the activation of macrophages by interferon- $\gamma$ to a microbicidal state, and the stimulation of IL-2 and IFN-gamma synthesis and consequent Th1 proliferation by IL-12 synthesized by dendritic cells. Macrophages and epithelial cells release IL-8 a potent chemokine for neutrophils. IL-6 is rapidly 
produced by many cell types in response to injury and also by activated macrophages. It is considered a proinflammatory cytokine implicated in the maintenance of inflammation and activation of Th17 lymphocytes, active in multiple chronic inflammatory diseases. Altogether, these complex interactions and inflammation can affect fertility and cause significant abdominal pain to patients with endometriosis.

To our knowledge, this systematic review and metaanalysis is the first one compiling all cytokines or interleukins ever evaluated in endometriosis patient fluids in relation to two main symptoms, infertility and pelvic pain. Our survey may have been affected by intrinsic study biases, as all evidence came from observational studies, which are at higher risk of bias than randomized, controlled trials. Therefore, the quality of the evidence may be reduced because the studies present several limitations, as for instance the way results and data information were presented in the papers (Fig. 2). Also, the IL-6 meta-analysis results, even though it showed higher expression in serum from endometriosis group, should be interpreted with caution, since it is derived from only two studies, with mild-degree heterogeneity and, concrete assumption and association made from these results are subjected to miscalculation.

Some points have to be addressed in future research. Among the first things that should be debated is endometriosis classification and the type of lesions that have to be considered and specified in the studies. Some authors use the term early stages, referring to stages I and II and advanced stages for stages III and IV; others classify endometriosis patients by the presence of peritoneal and endometriotic ovarian cysts. In addition, there is reference to superficial and deep nodules of the disease. According to the latest World Endometriosis Society consensus on the classification of endometriosis published in the beginning of 2017 (Johnson et al. 2017), endometriosis final classification include stage I (minimal), II (mild), III (moderate) and IV (severe). To conform to this classification, a series of grades that include observation of peritoneal and ovary lesions should be considered. The location of the lesion and its type should be described, and also whether it is superficial or deep and other individualities. Therefore, when comparing groups of patients, all these characteristics should be addressed and described in the article, especially when the comparison is among endometriosis groups.

Furthermore, the choice of an ideal control group to be compared with endometriosis will mostly depend on the hypothesis and the expected endpoint that is under consideration. Since endometriosis is a multifactorial disease and each stage or type of lesion is sometimes considered a different disease (Nisolle \& Donnez 1997), it is important to clarify what type of disease the researches are working on, describe each patient (comorbidities, use of hormone, therapies) and the selection criteria of all groups. Nevertheless, the establishment of a constant control group used in the studies or, at least, more homogeneous groups would be an important but quite hard purpose to accomplish. As observed, the control groups present in the studies considered in this review are widely different and possibly may have influenced the results. Examples of conditions of patients who were in the control groups are the following: pelvic inflammatory disease; pelvic disease; benign ovarian cyst; idiopathic infertility; tubal infertility; diagnostic laparoscopy for infertility; antisperm antibody investigation; assisted reproduction without endometriosis; uterine fibroids; leiomyoma; tubal ligation or reanastomosis.

Since the approach to the abovementioned studies are related to inflammatory conditions, the selection of control groups solely by absence of endometriotic lesions as the only inclusion criterion, disregarding other inflammatory diseases, may be the major factor for the non-significant, hard to interpret, findings of most studies. Crohn's diseases, for example, as well as other inflammatory diseases, are known to have cytokines involved in their pathogenesis (Guan \& Zhang 2017). Even stressed people can have in their body fluids deregulated cytokine levels (Clark et al. 2019). Because of the involvement of these molecules in a number of other pathological conditions, patients included in the control groups should be better analyzed and considered prior to their participation in endometriosis-related studies. The task is not simple since many of these other inflammatory diseases, as well as endometriosis, cannot be checked by a precise non-invasive diagnostic method.

Although the available evidence points to increased cytokine concentrations in endometriosis patients, it is still not clear if and how cytokines, especially interleukins, participate in the physiopathology of endometriosis-associated infertility and pelvic pain. Different fluids of the same patient may present different profiles of endometriosis-related molecules. In addition, the choice of an adequate control group is essential. Considering the potential use of cytokines as a future diagnostic or biomarker tool in endometriosis or for endometriosis-related infertility or pelvic pain, it is mandatory that investigators consider the correct choice of control groups, the endometriosis grade and the type of observed lesions and other inflammatory diseases that may obscure the results.

\section{Supplementary data}

This is linked to the online version of the paper at https://doi.org/10.1530/REP-18-0618.

\section{Declaration of interest}

The authors declare that there is no conflict of interest that could be perceived as prejudicing the impartiality of the research reported. 


\section{Funding}

This work was supported by the São Paulo Research Foundation (FAPESP-Fundação de Amparo à Pesquisa do Estado de São Paulo; grant number 2018/11042-0).

\section{Acknowledgements}

The authors would like to thank Dr Ises de Almeida Abrahamsohn for the support and for critically reading the manuscript.

\section{References}

Akoum A, Al-Akoum M, Lemay A, Maheux R \& Leboeuf M 2008 Imbalance in the peritoneal levels of interleukin 1 and its decoy inhibitory receptor type $\mathrm{II}$ in endometriosis women with infertility and pelvic pain. Fertility and Sterility 89 1618-1624. (https://doi.org/10.1016/j. fertnstert.2007.06.019)

Andreoli CG, Genro VK, Souza CA, Michelon T, Bilibio JP, Scheffel C \& Cunha-Filho JS 2011 T helper (Th)1, Th2, and Th17 interleukin pathways in infertile patients with minimal/mild endometriosis. Fertility and Sterility 95 2477-2480. (https://doi.org/10.1016/j. fertnstert.2011.02.019)

Andres Mde P, Podgaec S, Carreiro KB \& Baracat EC 2014 Endometriosis is an important cause of pelvic pain in adolescence. Revista da Associação Médica Brasileira 60 560-564. (https://doi.org/10.1590/18069282.60.06.015)

Arici A 2002 Local cytokines in endometrial tissue: the role of interleukin-8 in the pathogenesis of endometriosis. Annals of the New York Academy of Sciences 955 101-109; discussion 118, 396. (https://doi. org/10.1111/j.1749-6632.2002.tb02770.x)

Arici A, Matalliotakis I, Goumenou A, Koumantakis G, Vassiliadis S \& Mahutte NG 2003a Altered expression of interleukin-18 in the peritoneal fluid of women with endometriosis. Fertility and Sterility 80 889-894. (https://doi.org/10.1016/S0015-0282(03)01122-1)

Arici A, Matalliotakis I, Goumenou A, Koumantakis G, Vassiliadis S, Selam B \& Mahutte NG 2003b Increased levels of interleukin-15 in the peritoneal fluid of women with endometriosis: inverse correlation with stage and depth of invasion. Human Reproduction 18 429-432. (https:// doi.org/10.1093/humrep/deg083)

Arnold J, Vercellino GF, Chiantera V, Schneider A, Mechsner S \& Barcena de Arellano ML2013 Neuroimmunomodulatory alterations in non-lesional peritoneum close to peritoneal endometriosis. Neuroimmunomodulation 20 9-18. (https://doi.org/10.1159/000342163)

Buyalos RP, Watson JM \& Martinez-Maza O 1992 Detection of interleukin-6 in human follicular fluid. Fertility and Sterility 57 1230-1234. (https:// doi.org/10.1016/S0015-0282(16)55079-1)

Carvalho LF, Rossener R, Azeem A, Malvezzi H, Simões Abrão M \& Agarwal A 2013 From conception to birth: how endometriosis affects the development of each stage of reproductive life. Minerva Ginecologica 65 181-198.

Clark SM, Song C, Li X, Keegan AD \& Tonelli LH 2019 CD8+T cells promote cytokine responses to stress. Cytokine 113 256-264. (https:// doi.org/10.1016/j.cyto.2018.07.015)

Da Broi MG \& Navarro PA 2016 Oxidative stress and oocyte quality: ethiopathogenic mechanisms of minimal/mild endometriosis-related infertility. Cell and Tissue Research 364 1-7. (https://doi.org/10.1007/ s00441-015-2339-9)

Drosdzol-Cop A \& Skrzypulec-Plinta V 2012 Selected cytokines and glycodelin A levels in serum and peritoneal fluid in girls with endometriosis. Journal of Obstetrics and Gynaecology Research 38 1245-1253. (https://doi.org/10.1111/j.1447-0756.2012.01860.x)

Drosdzol-Cop A, Skrzypulec-Plinta V \& Stojko R 2012 Serum and peritoneal fluid immunological markers in adolescent girls with chronic pelvic pain. Obstetrical and Gynecological Survey 67 374-381. (https:// doi.org/10.1097/OGX.0b013e31825cb12b)

Eisenberg VH, Weil C, Chodick G \& Shalev V2018 Epidemiology of endometriosis: a large population-based database study from a healthcare provider with 2 million members. BJOG 125 55-62. (https:// doi.org/10.1111/1471-0528.14711)

Fairbanks F, Abrão MS, Podgaec S, Dias JA, de Oliveira RM \& Rizzo LV 2009 Interleukin-12 but not interleukin-18 is associated with severe endometriosis. Fertility and Sterility 91 320-324. (https://doi. org/10.1016/j.fertnstert.2007.11.060)

Furuya K, Murakami M, Makimura N, Matsuda H, Ikou K, Saito K, Kawakami Y, Shibazaki T, Fukui U, Mizumoto Y et al. 2003 Immunological and endocrinological studies on lymphocyte subpopulation and medical treatment for infertility in patients with endometriosis. Molecular and Cellular Endocrinology 202 195-199. (https://doi.org/10.1016/S03037207(03)00083-2)

Gazvani MR, Christmas S, Quenby S, Kirwan J, Johnson PM \& Kingsland CR 1998 Peritoneal fluid concentrations of interleukin-8 in women with endometriosis: relationship to stage of disease. Human Reproduction 13 1957-1961. (https://doi.org/10.1093/HUMREP/13.7.1957)

Glitz C, Souza CA, Rodini GP, Genro V, Bilibio JP, Senger M \& CunhaFilho JS 2009 Peritoneal and serum interleukin-18 levels are not increased in women with minimum or mild endometriosis. Brazilian Journal of Medical and Biological Research 42 1039-1043. (https://doi. org/10.1590/S0100-879X2009001100007)

Guan Q \& Zhang J 2017 Review article recent advances: the imbalance of cytokines in the pathogenesis of inflammatory bowel disease. Mediators of Inflammation 2017 4810258. (https://doi. org/10.1155/2017/4810258)

Gupta D, Hull ML, Fraser I, Miller L, Bossuyt PM, Johnson N \& Nisenblat V 2016 Endometrial biomarkers for the non-invasive diagnosis of endometriosis (Review). Cochrane Library 4 1-231. (https://doi. org/10.1002/14651858.CD012165.Copyright)

Harada T, Yoshioka H, Yoshida S, Iwabe T, Onohara Y, Tanikawa M \& Terakawa N 1997 Increased interleukin-6 levels in peritoneal fluid of infertile patients with active endometriosis. American Journal of Obstetrics and Gynecology 176 593-597. (https://doi.org/10.1016/ S0002-9378(97)70553-2)

Harada T, Iwabe T \& Terakawa N 2001 Role of cytokines in endometriosis. Fertility and Sterility 76 1-10. (https://doi.org/10.1016/S00150282(01)01816-7)

Haydardedeoglu B \& Zeyneloglu HB 2015 The impact of endometriosis on fertility. Women's Health 11 619-623. (https://doi.org/10.2217/ whe.15.48)

Hsu AL, Townsend PM, Oehninger S \& Castora FJ 2015 Endometriosis may be associated with mitochondrial dysfunction in cumulus cells from subjects undergoing in vitro fertilization-intracytoplasmic sperm injection, as reflected by decreased adenosine triphosphate production. Fertility and Sterility 103 347.e1-352.e1. (https://doi.org/10.1016/j. fertnstert.2014.11.002)

Izumi G, Koga K, Takamura M, Makabe T, Satake E, Takeuchi A, Taguchi A, Urata Y, Fujii T \& Osuga Y 2018 Involvement of immune cells in the pathogenesis of endometriosis. Journal of Obstetrics and Gynaecology Research 44 191-198. (https://doi.org/10.1111/jog.13559)

Jiang L, Yan Y, Liu Z \&Wang Y 2016 Inflammation and endometriosis. Frontiers in Bioscience 21 941-948. (https://doi.org/10.1002/9781444398519. ch24)

Johnson NP, Hummelshoj L, Adamson GD, Keckstein J, Taylor HS, Abrao MS, Bush D, Kiesel L, Tamimi R, Sharpe-Timms KL et al. 2017 World endometriosis society consensus on the classification of endometriosis. Human Reproduction 32 315-324. (https://doi. org/10.1093/humrep/dew293)

Kondera-Anasz Z, Sikora J, Mielczarek-Palacz A \& Jońca M 2005 Concentrations of interleukin (IL)- $1 \alpha$, IL- 1 soluble receptor type II (IL1 sRII) and IL-1 receptor antagonist (IL-1 Ra) in the peritoneal fluid and serum of infertile women with endometriosis. European Journal of Obstetrics, Gynecology, and Reproductive Biology 123 198-203. (https://doi.org/10.1016/j.ejogrb.2005.04.019)

Kvaskoff M, Mu F, Terry KL, Harris HR, Poole EM, Farland L \& Missmer SA 2015 Endometriosis: A high-risk population for major chronic diseases? Human Reproduction Update 21 500-516. (https://doi.org/10.1093/ humupd/dmv013)

Liu Y, Luo L \& Zhao H 2000 Changes of cytokines levels in peritoneal fluids of patients with endometriosis and its effect on reproductive activity. Journal of Tongji Medical University 20 163-165. (https://doi. org/10.1007/BF02887062) 
Long X, Ye Y, Zhang L, Liu P, Yu W, Wei F, Ren X \& Yu J 2016 IL-8, a novel messenger to cross-link inflammation and tumor EMT via autocrine and paracrine pathways (Review). International Journal of Oncology 48 5-12. (https://doi.org/10.3892/ijo.2015.3234)

Lubberts E 2015 The IL-23-IL-17 axis in inflammatory arthritis. Nature Reviews. Rheumatology 11 415-429. (https://doi.org/10.1038/ nrrheum.2015.53)

Malhotra N, Karmakar D, Tripathi V, Luthra K \& Kumar S 2012 Correlation of angiogenic cytokines-leptin and IL-8 in stage, type and presentation of endometriosis. Gynecological Endocrinology 28 224-227. (https://doi.or g/10.3109/09513590.2011.593664)

Malvezzi H, Da Broi MG, Meola J, Rosa-E-Silva JC, Ferriani RA \& Navarro PA 2018 Peritoneal fluid of women with endometriosis reduces SOD1 in bovine oocytes in vitro maturation. Cell and Tissue Research 372 621-628. (https://doi.org/10.1007/s00441-018-2805-2)

Manero MG \& Alcazar JL 2010 Interleukin-8 serum levels do not correlate with pelvic pain in patients with ovarian endometriomas. Fertility and Sterility 94 450-452. (https://doi.org/10.1016/j.fertnstert.2009.03.074)

McLaren J, Dealtry G, Prentice A, Charnock-Jones DS \& Smith SK 1997 Decreased levels of the potent regulator of monocyte/macrophage activation, interleukin-13, in the peritoneal fluid of patients with endometriosis. Human Reproduction 12 1307-1310. (https://doi. org/10.1093/humrep/12.6.1307)

Michaud N, Al-Akoum M, Gagnon G, Girard K, Blanchet P, Rousseau JA \& Akoum A 2011 Decreased concentrations of soluble interleukin-1 receptor accessory protein levels in the peritoneal fluid of women with endometriosis. Journal of Reproductive Immunology 92 68-73. (https:// doi.org/10.1016/j.jri.2011.08.001)

Mikolajczyk M, Wirstlein P \& Skrzypczak J 2006 Leukaemia inhibitory factor and interleukin 11 levels in uterine flushings of infertile patients with endometriosis. Human Reproduction 21 3054-3058. (https://doi. org/10.1093/humrep/del225)

Miller JE, Ahn SH, Monsanto SP, Khalaj K, Koti M \& Tayade C 2017 Implications of immune dysfunction on endometriosis associated infertility. Oncotarget 8 7138-7147. (https://doi.org/10.18632/ oncotarget.12577)

Musso T, Calosso L, Zucca M, Millesimo M, Puliti M, BulfonePaus S, Merlino C, Savoia D, Cavallo R, Ponzi AN et al. 1998 Interleukin-15 activates proinflammatory and antimicrobial functions in polymorphonuclear cells. Infection and Immunity 66 2640-2647)

Nesbitt-Hawes E \& Ledger W 2004 Endometriosis and infertility. Fertility and Sterility 86 (Supplement 4) S156-S160. (https://doi.org/10.1007/9781-4471-4953-8_3)

Nisenblat V, Bossuyt PM, Shaikh R, Farquhar C, Jordan V, Scheffers CS, Mol BW, Johnson N \& Hull ML 2016 Blood biomarkers for the noninvasive diagnosis of endometriosis. Cochrane Database of Systematic Reviews 5 CD012179. (https://doi.org/10.1002/14651858.CD012179)

Nisolle M \& Donnez J 1997 Peritoneal endometriosis, ovarian endometriosis, and adenomyotic nodules of the rectovaginal septum are three different entities. Fertility and Sterility 68 585-596. (https://doi. org/10.1016/S0015-0282(97)00191-X)

Oku H, Tsuji Y, Kashiwamura SI, Adachi S, Kubota A, Okamura H \& Koyama K 2004 Role of IL-18 in pathogenesis of endometriosis. Human Reproduction 19 709-714. (https://doi.org/10.1093/humrep/deh108)

Pellicer A, Albert C, Mercader A, Bonilla-Musoles F, Remohí J \& Simón C 1998 The follicular and endocrine environment in women with endometriosis: local and systemic cytokine production. Fertility and Sterility 70 425-431. (https://doi.org/10.1016/S0015-0282(98)00204-0)

Podgaec S, Rizzo LV, Fernandes LF, Baracat EC \& Abrao MS 2012 CD4+CD25highFoxp3+ cells increased in the peritoneal fluid of patients with endometriosis. American Journal of Reproductive Immunology 68 301-308. (https://doi.org/10.1111/j.1600-0897.2012.01173.x)

Raphael I, Nalawade S, Eagar TN \& Forsthuber TG 2015 T cell subsets and their signature cytokines in autoimmune and inflammatory diseases. Cytokine 74 5-17. (https://doi.org/10.1016/j.cyto.2014.09.011)

Rapkin A, Morgan M, Bonpane C \& Martinez-Maza O 2000 Peritoneal fluid interleukin-6 in women with chronic pelvic pain. Fertility and Sterility 74 325-328. (https://doi.org/10.1016/S0015-0282(00)00653-1)

Santanam N, Murphy AA \& Parthasarathy S 2002 Macrophages, oxidation, and endometriosis. Annals of the New York Academy of Sciences 955 183-198; discussion 19-200, 396-406. (https://doi. org/10.1111/j.1749-6632.2002.tb02779.x)
Santulli P, Borghese B, Chouzenoux S, Vaiman D, Borderie D, Streuli I, Goffinet F, de Ziegler D, Weill B, Batteux F et al. 2012 Serum and peritoneal interleukin-33 levels are elevated in deeply infiltrating endometriosis. Human Reproduction 27 2001-2009. (https://doi. org/10.1093/humrep/des154)

Sikora J, Smycz-Kubańska M, Mielczarek-Palacz A \& Kondera-Anasz Z 2017 Abnormal peritoneal regulation of chemokine activation-the role of IL-8 in pathogenesis of endometriosis. American Journal of Reproductive Immunology 77 1-8. (https://doi.org/10.1111/aji.12622)

Singh AK, Dutta M, Chattopadhyay R, Chakravarty B \& Chaudhury K 2016 Intrafollicular interleukin-8, interleukin-12, and adrenomedullin are the promising prognostic markers of oocyte and embryo quality in women with endometriosis. Journal of Assisted Reproduction and Genetics 33 1363-1372. (https://doi.org/10.1007/s10815-016-0782-5)

Skrzypczak J, Szczepańska M, Puk E, Kamieniczna M \& Kurpisz M 2005 Peritoneal fluid cytokines and sICAM-1 in minimal endometriosis: search for discriminating factors between infertility and/or endometriosis. European Journal of Obstetrics and Gynecology and Reproductive Biology 122 95-103. (https://doi.org/10.1016/j.ejogrb.2004.11.044)

Slim K, Nini E, Forestier D, Kwiatkowski F, Panis Y \& Chipponi J 2003 Methodological index for non-randomized studies (Minors): development and validation of a new instrument. ANZ Journal of Surgery 73 712-716. (https://doi.org/10.1046/j.1445-2197.2003.02748.x)

Stilley JAW, Birt JA \& Sharpe-Timms KL 2012 Cellular and molecular basis for endometriosis-associated infertility. Cell and Tissue Research 349 849-862. (https://doi.org/10.1007/s00441-011-1309-0)

Suen JL, Chang Y, Chiu PR, Hsieh TH, Hsi E, Chen YC, Chen YF \& Tsai EM 2014 Serum level of IL-10 is increased in patients with endometriosis, and IL-10 promotes the growth of lesions in a murine model. American Journal of Pathology 184 464-471. (https://doi.org/10.1016/j. ajpath.2013.10.023)

Symons LK, Miller JE, Kay VR, Marks RM, Liblik K, Koti M \& Tayade C 2018 The immunopathophysiology of endometriosis. Trends in Molecular Medicine 24 748-762. (https://doi.org/10.1016/j.molmed.2018.07.004)

Ueki M, Tsurunaga T, Ushiroyama T \& Ueda M 1994 Macrophage activation factors and cytokines in peritoneal fluid from patients with endometriosis. Asia-Oceania Journal of Obstetrics and Gynaecology 20 427-431. (https://doi.org/10.1111/j.1447-0756.1994.tb00492.x)

Valentine JC, Pigott TD \& Rothstein HR 2010 How many studies do you need?: A primer on statistical power for meta-analysis. Journal of Educational and Behavioral Statistics 35 215-247. (https://doi. org/10.3102/1076998609346961)

Velasco I, Acién P, Campos A, Acién MI \& Ruiz-Maciá E 2010 Interleukin-6 and other soluble factors in peritoneal fluid and endometriomas and their relation to pain and aromatase expression. Journal of Reproductive Immunology 84 199-205. (https://doi.org/10.1016/j.jri.2009.11.004)

Vercellini P, Viganò P, Somigliana E \& Fedele L 2014 Endometriosis: pathogenesis and treatment. Nature Reviews Endocrinology 10 261-275. (https://doi.org/10.1038/nrendo.2013.255)

Wu G, Bersinger NA, Mueller MD \& von Wolff M 2017 Intrafollicular inflammatory cytokines but not steroid hormone concentrations are increased in naturally matured follicles of women with proven endometriosis. Journal of Assisted Reproduction and Genetics 34 357-364. (https://doi.org/10.1007/s10815-016-0865-3)

Wunder DM, Mueller MD, Birkhäuser MH \& Bersinger NA 2006 Increased ENA-78 in the follicular fluid of patients with endometriosis. Acta obstetricia et gynecologica Scandinavica 85 336-342. (https://doi. org/10.1080/00016340500501715)

Zhang X, Xu H, Lin J, Qian Y \& Deng L 2005 Peritoneal fluid concentrations of interleukin-17 correlate with the severity of endometriosis and infertility of this disorder. BJOG 112 1153-1155. (https://doi.org/10.1111/j.14710528.2005.00639.x)

Received 10 December 2018

First decision 14 March 2019

Revised manuscript received 20 March 2019

Accepted 1 April 2019 